Reform des Bundeswahlrechts

\section{Fiasko droht!}

Das Erschrecken über die hohe Zahl an Bundestagsabgeordneten war deutlich vernehmbar. Statt der gesetzlichen Zahl von 598 Abgeordneten entsandten die Wähler*innen bei der jüngsten Bundestagswahl 709 Abgeordnete in den Deutschen Bundestag. Doch eine grundlegende Reform ist gescheitert, vorerst, möchte man hoffend hinzufügen. Der Bundestag konnte sich am 3. Juli 2020 nicht auf eine Reform des Bundeswahlrechts verständigen. Der einzige Vorschlag von FDP, Linken und Grünen, der auf eine Verringerung der Wahlkreise zielt, kam nicht zur Abstimmung. Ein weiterer Vorschlag wurde nicht eingebracht.

Offensichtlich unstrittig ist, dass weiterhin das Prinzip der Verhältniswahl die Zusammensetzung des Bundestags bestimmen soll. Am dualen Prinzip aus direkt gewählten Abgeordneten und Abgeordneten aus den Landeslisten soll ebenfalls festgehalten werden. Die SPD favorisiert grundsätzlich den Status quo, will aber nach dem Wahlgang und vor der Konstituierung eine symmetrische Stauchung des Parlaments. Mit dem Vorschlag des Fraktionsvorsitzenden der CDU/CSU Ende Juni kamen neue Optionen ins Spiel. So wurde offenbar erwogen, dass einige direkt gewählte Abgeordnete trotz ihrer Wahl nicht in den Bundestag einziehen sollen. Damit sollten Ausgleichs- und Überhangmandate reduziert werden. Auch eine moderate Verringerung der 299 Wahlkreise wurde in Erwägung gezogen. Offenbar hatte es aber schon innerhalb der Koalition nicht für einen Kompromiss gereicht.

Immerhin fokussieren die meisten Vorschläge auf die Bestimmung der direkt gewählten Abgeordneten. Wollte man neben dem politischen Geschacher einen wissenschaftlich basierten Vorschlag unterbreiten, so würde man genau an dieser Stelle sinnvoll ansetzen können. Mit der Erststimme werden nach dem Prinzip der relativen Mehrheitswahl die direkt gewählten Abgeordneten bestimmt. Weil sie aktuell überwiegend aus dem Lager der Mehrheitspartei kommen, besteht ein hoher Bedarf an Ausgleichsmandaten, was zur Vergrößerung des Parlaments führt. Die relative Mehrheitswahl (plurality rule) ist seit langem der Wissenschaft, insbesondere der Social-Choice-Theorie, ein Dorn im Auge. Aus dieser Wahl kommt nicht zwingend diejenige Kandidatin als Siegerin hervor, die gegenüber allen anderen Kandidaten aus Sicht

(C) Der/die Autor(en) 2020. Open Access: Dieser Artikel wird unter der Creative Commons Namensnennung 4.0 International Lizenz (https:// creativecommons.org/licenses/by/4.0/deed.de) veröffentlicht.

Open Access wird durch die ZBW - Leibniz-Informationszentrum Wirtschaft gefördert. der Mehrheit der Wähler*innen vorgezogen wird (Condorcet-Siegerin), sondern eher jener, der auf ein günstiges Kandidatenumfeld trifft. Dabei sollte die Wahl unabhängig von irrelevanten Alternativen sein, wie Arrow es als ein demokratisches Prinzip bezeichnete. Seit Jahrzehnten besteht deshalb eine unumstrittene axiomatische Überlegenheit der einfachen Mehrheitswahl gegenüber der relativen Mehrheitswahl, wie die Arbeiten von May (1952) bis Dasgupta und Maskin (2014) zeigen. Erst jüngst warben Amartya Sen und Eric Maskin für die Einführung der einfachen Mehrheitswahl in den US-Vorwahlen und zeigten, dass Trump unter diesem überlegenen Wahlrecht nicht Präsident geworden wäre.

Angewendet auf die Bundestagswahl würde die Heterogenität der direkt gewählten Abgeordneten zunehmen, was wegen des damit verbundenen geringeren Ausgleichsbedarfs zu einer Verkleinerung des Bundestags führen würde. Die einfache Mehrheitswahl hätte aber noch einen zweiten Effekt auf die Größe des Bundestags. Es würde nicht in jedem Wahlkreis einen direkt gewählten Abgeordneten geben, denn nicht in jedem Wahlkreis gibt es eine Condorcet-Siegerin, die jeden anderen möglichen Kandidaten in einer paarweisen Abstimmung besiegt. Wenn es eine solche nicht gibt, dann spricht auch nichts dafür, dass dennoch eine Person als direkt gewählte Abgeordnete in den Bundestag einzieht. Eine Reduzierung der direkt gewählten Abgeordneten wäre also die Folge. Das zieht im Ergebnis die demokratische Opposition wie auch offenbar Teile von CDU/CSU und SPD in Erwägung. Nur würde dies nicht durch größere Wahlkreise erreicht oder gar dadurch, dass Wahlsieger keinen Zutritt zum Parlament erhalten (was nach einer langwierigen verfassungsrechtlichen Auseinandersetzung klingt), sondern durch ein im Grunde unumstritten überlegenes Wahlrecht. Es wäre im Bundeswahlrecht noch nicht einmal viel zu ändern. Im Wesentlichen muss den Wähler*innen erlaubt werden, ihre Präferenzordnung bezüglich der Kandidat*innen offenlegen zu können, statt, wie bislang, nur ihre erste Präferenz.

Die Zeit ist knapp. Will man substanziell ein Scheitern der Bundeswahlrechtsreform vermeiden, dann sind wissenschaftlich fundierte Vorschläge nötig. Demokratische Wahlen sind ein unschätzbar hohes Gut. Sie sollten deshalb die Kriterien der Anonymität, der Neutralität und der „positive responsiveness“ erfüllen. Die relative Mehrheitswahl tut dies bekanntlich nicht, die einfache Mehrheitswahl kann diese Kriterien erfüllen. Die Social-ChoiceTheorie kann zur Wahlrechtsreform einen wesentlichen Beitrag leisten. Sie sollte es auch!

Salvatore Barbaro Johannes-Gutenberg-Universität Mainz sbarbaro@uni-mainz.de 